\title{
Energy levels and radiative rates for inner shell transitions of $\mathrm{Fe} \mathrm{xVI}^{\star}$
}

\author{
K. M. Aggarwal and F. P. Keenan
}

\begin{abstract}
Astrophysics Research Centre, School of Mathematics and Physics, Queen's University, Belfast BT7 1NN, Northern Ireland, UK e-mail: K.Aggarwal@qub.ac.uk
\end{abstract}

Received 11 May 2006 / Accepted 16 November 2006

\section{ABSTRACT}

\begin{abstract}
Aims. In this paper we report calculations for energy levels and radiative rates for transitions in Fe XVI. Methods. The General purpose Relativistic Atomic Structure Package (GRASP) has been adopted for the calculations of energy levels and radiative rates.

Results. Energies for the lowest 134 levels among the $\left(1 \mathrm{~s}^{2} 2 \mathrm{~s}^{2}\right) 2 \mathrm{p}^{6} 3 \ell, 2 \mathrm{p}^{5} 3 \mathrm{~s}^{2}, 2 \mathrm{p}^{5} 3 \mathrm{~s} 3 \mathrm{p}, 2 \mathrm{p}^{5} 3 \mathrm{~s} 3 \mathrm{~d}, 2 \mathrm{p}^{5} 3 \mathrm{p} 3 \mathrm{~d}$, and $2 \mathrm{p}^{5} 3 \mathrm{p}^{2}$ configurations of Fe XVI are reported. Additionally, radiative rates, oscillator strengths, and line strengths are reported for all electric dipole (E1), magnetic dipole (M1), electric quadrupole (E2), magnetic quadrupole (M2), and electric octupole (E3) transitions among these levels. Comparisons are made with the available results in the literature, and the accuracy of the present results is assessed. Finally, lifetimes for all excited levels are also listed, although no measurements are presently available with which to compare these.
\end{abstract}

Key words. atomic data - atomic processes

\section{Introduction}

In a recent paper (Aggarwal \& Keenan 2006) we reported energy levels, radiative rates, collision strengths, and excitation rate coefficients for transitions among 39 levels of the $\left(1 s^{2} 2 s^{2} 2 p^{6}\right) n l$; $n \leq 7, \ell \leq 4$ configurations of Fe XVI. However, many lines of Fe XVI in the 15-17 $\AA$ X-ray range are frequently observed in astrophysical and laboratory plasmas, and some of these arise from the inner (L) shell excitation of Fe XVI, such as: $2 \mathrm{p}^{6} 3 \mathrm{~s}+\mathrm{e}^{-} \rightarrow$ $2 \mathrm{p}^{5} 3 \ell 3 \ell^{\prime}$ (see, for example, Dere et al. 2001). These emission lines particularly arise at comparatively higher temperatures, and hence atomic data for such transitions are also useful in fusion plasmas. Therefore, in this paper we focus on the inner shell electron impact excitation of Fe XVI.

Earlier calculations for this ion have been performed by Cornille et al. (1994) and Bautista (2000). Cornille et al. adopted the SuperStructure (SS) code of Eissner et al. (1974) for the generation of wavefunctions, and the distorted-wave (DW) code of Eissner \& Seaton (1972) for the computations of collision strengths $(\Omega)$. They included 44 levels of the $2 p^{6} 3 s, 2 p^{5} 3 s^{2}$, $2 p^{5} 3 \mathrm{~s} 3 \mathrm{p}$ and $2 \mathrm{p}^{5} 3 \mathrm{~s} 3 \mathrm{~d}$ configurations in their calculations, and reported values of $\Omega$ at a single energy of $60 \mathrm{Ryd}$. The main deficiency of their work is that they neither considered the variation of $\Omega$ with energy, nor included the contribution of resonances in the determination of excitation rates. Both of these deficiencies were removed in the subsequent work of Bautista, who adopted a semi-relativistic $R$-matrix approach (Berrington et al. 1995). In his calculations, he included $59 L S$ terms of the $2 \mathrm{p}^{6} 3 \ell, 2 \mathrm{p}^{5} 3 \mathrm{~s}^{2}$, $2 \mathrm{p}^{5} 3 \mathrm{~s} 3 \mathrm{p}, 2 \mathrm{p}^{5} 3 \mathrm{~s} 3 \mathrm{~d}, 2 \mathrm{p}^{5} 3 \mathrm{p} 3 \mathrm{~d}$, and $2 \mathrm{p}^{5} 3 \mathrm{p}^{2}$ configurations, and obtained results for fine-structure transitions through an algebraic transformation. In this process, he also included the relativistic effects through term coupling coefficients (TCC). Since Fe XVI is a moderately heavy ion $(Z=26)$, both configuration interaction

* Tables 2, 3 and 5 are only available in electronic form at the CDS via anonymous ftp to cdsarc.u-strasbg.fr $(130.79 .128 .5)$ or via

http://cdsweb.u-strasbg.fr/cgi-bin/qcat? J/A+A/463/399
(CI) and relativistic effects are supposed to be important in the determination of wavefunctions, and subsequently of $\Omega$. He observed that the contribution of resonances has enhanced the effective collision strengths $(\Upsilon)$ by up to three orders of magnitude, depending on the temperature, and the relativistic effects have enhanced these by several orders of magnitude, for some of the transitions. Since both of these observed enhancements are unusually high, one of our aims is to assess the accuracy of his reported results, apart from making other improvements.

In the present work, we include 134 levels among the $\left(1 \mathrm{~s}^{2} 2 \mathrm{~s}^{2}\right) 2 \mathrm{p}^{6} 3 \ell, 2 \mathrm{p}^{5} 3 \mathrm{~s}^{2}, 2 \mathrm{p}^{5} 3 \mathrm{~s} 3 \mathrm{p}, 2 \mathrm{p}^{5} 3 \mathrm{~s} 3 \mathrm{~d}, 2 \mathrm{p}^{5} 3 \mathrm{p} 3 \mathrm{~d}$, and $2 \mathrm{p}^{5} 3 \mathrm{p}^{2}$ configurations of Fe XVI. Additionally, our approach is fully relativistic, as we employ the GRASP (General purpose Relativistic Atomic Structure Package) code of Dyall et al. (1989) for the generation of wavefunctions, and the Dirac Atomic $R$-matrix Code (DARC) of Norrington \& Grant (2007) for computations of $\Omega$, and subsequently of $\Upsilon$. However, it may be noted that the $2 p^{5} 3 d^{2}$ configuration has been deliberately excluded from the calculations, because it generates 45 levels and all of which lie above the lowest 134 already included. The same argument applies to other higher configurations, such as $2 \mathrm{p}^{5} 3 \ell 4 \ell$ and $2 \mathrm{p}^{5} 4 \ell^{2}$. Since all of these configurations give rise to energy levels above the 134 levels included presently, their inclusion in a calculation will not significantly affect the results for energy levels and/or the radiative rates among the lowest 134 levels. For example, a calculation performed with the additional inclusion of the $2 \mathrm{p}^{5} 3 \mathrm{~d}^{2}, 2 \mathrm{p}^{5} 3 \ell 4 \ell$ and $2 \mathrm{p}^{5} 4 \ell^{2}$ configurations, resulting in a total of 1201 levels, yields energy levels within $1 \mathrm{eV}$ (or equivalently within $0.1 \%$ ) with those listed in Table 1 . Therefore, their specific inclusion in a calculation may affect the results of effective collision strengths (at higher electron temperatures), due to the inclusion of resonances arising from the higher levels, but will not affect the reported results of energy levels and/or the radiative rates. Furthermore, the calculations for $\Omega$ (and subsequently for $\Upsilon$ ) are computationally demanding, as the size of the Hamiltonian matrix is large (13 330), and hence will take a considerably long time to conclude. Therefore, in this 
Table 1. Energy levels (in Ryd) and mixing coefficients of Fe XVI.

\begin{tabular}{|c|c|c|c|c|c|c|c|}
\hline Index & Configuration & Level & NIST & GRASP $^{a}$ & GRASP $^{b}$ & FAC $^{c}$ & Mixing coefficients $^{d}$ \\
\hline 1 & $2 p^{6} 3 s$ & ${ }^{2} \mathrm{~S}_{1 / 2}$ & 0.00000 & 0.00000 & 0.00000 & 0.00000 & $0.999(1)$ \\
\hline 2 & $2 p^{6} 3 p$ & ${ }^{2} \mathrm{P}^{\circ}{ }_{1 / 2}$ & 2.52598 & 2.56393 & 2.56618 & 2.54757 & $0.999(2)$ \\
\hline 3 & $2 p^{6} 3 p$ & ${ }^{2} \mathrm{P}_{3 / 2}^{\circ}$ & 2.71688 & 2.75844 & 2.75486 & 2.73706 & $0.999(3)$ \\
\hline 4 & $2 p^{6} 3 d$ & ${ }^{2} \mathrm{D}_{3 / 2}$ & 6.15562 & 6.20499 & 6.19591 & 6.16949 & $0.999(4)$ \\
\hline 5 & $2 p^{6} 3 d$ & ${ }^{2} \mathrm{D}_{5 / 2}$ & 6.18209 & 6.23332 & 6.22081 & 6.19510 & $0.999(5)$ \\
\hline 6 & $2 p^{5} 3 s^{2}$ & ${ }^{2} \mathrm{P}_{3 / 2}^{\circ}$ & 52.60745 & 52.36353 & 52.31794 & 52.41746 & $0.985(6)$ \\
\hline 7 & $2 p^{5} 3 s^{2}$ & ${ }^{2} \mathrm{P}_{1 / 2}^{\circ}$ & 53.51871 & 53.30895 & 53.24028 & 53.33762 & $0.985(7)$ \\
\hline 8 & $2 p^{5} 3 s 3 p$ & ${ }^{4} \mathrm{~S}_{3 / 2}$ & & 54.13058 & 54.08374 & 54.17709 & $-0.886(8), 0.431(14)$ \\
\hline 9 & $2 p^{5} 3 s 3 p$ & ${ }^{4} \mathrm{D}_{5 / 2}$ & 54.51199 & 54.37708 & 54.32940 & 54.42538 & $0.828(9),-0.416(13)$ \\
\hline 10 & $2 p^{5} 3 s 3 p$ & ${ }^{4} \mathrm{D}_{7 / 2}^{5 / 2}$ & & 54.43349 & 54.38145 & 54.47626 & $0.998(10)$ \\
\hline 11 & $2 p^{5} 3 s\left({ }^{1} \mathrm{P}\right) 3 \mathrm{p}$ & ${ }^{2} \mathrm{D}_{3 / 2}$ & 54.79442 & 54.43554 & 54.38938 & 54.48851 & $0.676(17), 0.536(11)$ \\
\hline 12 & $2 \mathrm{p}^{5} 3 \mathrm{~s}\left({ }^{1} \mathrm{P}\right) 3 \mathrm{p}$ & ${ }^{2} \mathrm{P}_{1 / 2}^{3 / 2}$ & 54.68514 & 54.56418 & 54.52045 & 54.61812 & $0.582(12),-0.541(16), 0.478(18)$ \\
\hline 13 & $2 p^{5} 3 s 3 p$ & ${ }^{4} \mathrm{P}_{5 / 2}$ & & 54.67382 & 54.62489 & 54.72388 & $0.810(13), 0.481(21)$ \\
\hline 14 & $2 p^{5} 3 s 3 p$ & ${ }^{4} \mathrm{P}_{3 / 2}$ & 55.55084 & 54.67609 & 54.62869 & 54.72922 & $0.543(11), 0.507(14), 0.431(22)$ \\
\hline 15 & $2 p^{5} 3 s\left({ }^{1} P\right) 3 p$ & ${ }^{2} \mathrm{~S}_{1 / 2}$ & 55.05876 & 54.89407 & 54.84442 & 54.93897 & $0.646(15),-0.537(18)$ \\
\hline 16 & $2 p^{5} 3 s 3 p$ & ${ }^{4} \mathrm{D}_{1 / 2}$ & 55.35947 & 55.28057 & 55.21306 & 55.30909 & $0.797(16), 0.453(12)$ \\
\hline 17 & $2 p^{5} 3 s 3 p$ & ${ }^{4} \mathrm{D}_{3 / 2}$ & 55.48705 & 55.37720 & 55.30739 & 55.40431 & $0.696(17),-0.430(11)$ \\
\hline 18 & $2 p^{5} 3 s 3 p$ & ${ }^{4} \mathrm{P}_{1 / 2}$ & 55.48705 & 55.39547 & 55.33684 & 55.42765 & $0.648(18), 0.545(15)$ \\
\hline 19 & $2 p^{5} 3 s\left({ }^{3} P\right) 3 p$ & ${ }^{2} \mathrm{D}_{5 / 2}$ & 54.79442 & 55.49867 & 55.44467 & 55.52949 & $-0.908(19)$ \\
\hline 20 & $2 \mathrm{p}^{5} 3 \mathrm{~s}\left({ }^{3} \mathrm{P}\right) 3 \mathrm{p}$ & ${ }^{2} \mathrm{P}_{3 / 2}$ & 55.85156 & 55.50353 & 55.43583 & 55.52862 & $0.663(14),-0.521(20)$ \\
\hline 21 & $2 p^{5} 3 s\left({ }^{1} \mathrm{P}\right) 3 \mathrm{p}$ & ${ }^{2} \mathrm{D}_{5 / 2}$ & 55.67842 & 55.57474 & 55.50469 & 55.60006 & $-0.788(21), 0.464(9)$ \\
\hline 22 & $2 p^{5} 3 s\left({ }^{1} P\right) 3 p$ & ${ }^{2} \mathrm{P}_{3 / 2}$ & & 55.63602 & 55.58697 & 55.67262 & $-0.642(22), 0.561(20),-0.418(24)$ \\
\hline 23 & $2 p^{5} 3 s\left({ }^{3} P\right) 3 p$ & ${ }^{2} \mathrm{P}_{1 / 2}$ & & 56.31892 & 56.25643 & 56.34105 & $0.737(23),-0.420(12),-0.419(15)$ \\
\hline 24 & $2 p^{5} 3 s\left({ }^{3} P\right) 3 p$ & ${ }^{2} \mathrm{D}_{3 / 2}$ & 56.65347 & 56.48207 & 56.40948 & 56.48980 & $0.761(24),-0.412(11)$ \\
\hline 25 & $2 p^{5} 3 s\left({ }^{3} P\right) 3 p$ & ${ }^{2} \mathrm{~S}_{1 / 2}$ & 57.10911 & 57.04715 & 56.99370 & 57.07898 & $0.894(25)$ \\
\hline 26 & $2 p^{5} 3 p^{2}$ & ${ }^{4} \mathrm{P}_{3 / 2}^{\circ}$ & & 57.11374 & 57.06488 & 57.15728 & $-0.579(26), 0.471(30)$ \\
\hline 27 & $2 p^{5} 3 p^{2}\left({ }^{1} D\right)$ & ${ }^{2} \mathrm{P}_{1 / 2}^{\circ}$ & & 57.11721 & 57.06704 & 57.16091 & $0.708(27),-0.447(63)$ \\
\hline 28 & $2 p^{5} 3 p^{2}$ & ${ }^{4} \mathrm{P}^{\circ}{ }_{5 / 2}$ & & 57.18875 & 57.13944 & 57.22878 & $0.861(28),-0.457(35)$ \\
\hline 29 & $2 p^{5} 3 p^{2}$ & ${ }^{2} \mathrm{~F}^{\circ} 7 / 2$ & & 57.20144 & 57.14806 & 57.24572 & $-0.828(29)$ \\
\hline 30 & $2 p^{5} 3 p^{2}\left({ }^{1} D\right)$ & ${ }^{2} \mathrm{P}_{3 / 2}^{\circ}$ & & 57.27558 & 57.22768 & 57.32069 & $-0.517(26),-0.415(30)$ \\
\hline 31 & $2 p^{5} 3 p^{2}\left({ }^{1} D\right)$ & ${ }^{2} \mathrm{D}^{\circ}{ }_{5 / 2}$ & & 57.40167 & 57.35118 & 57.44791 & $-0.624(31), 0.442(42)$ \\
\hline 32 & $2 p^{5} 3 p^{2}\left({ }^{3} P\right)$ & ${ }^{2} \mathrm{D}^{\circ}{ }_{3 / 2}$ & & 57.43847 & 57.39127 & 57.48571 & $0.783(32), 0.420(43)$ \\
\hline 33 & $2 p^{5} 3 p^{2}$ & ${ }^{4} \mathrm{P}_{1 / 2}^{\circ}$ & & 57.45866 & 57.40547 & 57.49659 & $-0.765(33), 0.490(45)$ \\
\hline 34 & $2 p^{5} 3 p^{2}$ & ${ }^{4} \mathrm{D}^{\circ}{ }_{7 / 2}$ & & 57.47691 & 57.42105 & 57.51299 & $0.944(34)$ \\
\hline 35 & $2 p^{5} 3 p^{2}$ & ${ }^{4} \mathrm{D}^{\circ}{ }_{5 / 2}^{1 / 2}$ & & 57.49089 & 57.43729 & 57.53061 & $0.591(51), 0.555(35), 0.404(28)$ \\
\hline 36 & $2 p^{5} 3 p^{2}$ & ${ }^{4} \mathrm{D}^{\circ}{ }_{1 / 2}^{0 / 2}$ & & 57.93073 & 57.87458 & 57.96637 & $0.788(36),-0.440(45)$ \\
\hline 37 & $2 p^{5} 3 p^{2}\left({ }^{1} D\right)$ & ${ }^{2} \mathrm{D}^{\circ}{ }_{3 / 2}^{1 / 2}$ & & 57.97229 & 57.91773 & 58.01218 & $0.617(48),-0.465(37)$ \\
\hline 38 & $2 p^{5} 3 s 3 d$ & ${ }^{4} \mathrm{P}_{1 / 2}^{\circ}{ }_{1 / 2}$ & & 57.98889 & 57.93888 & 58.03972 & $-0.979(38)$ \\
\hline 39 & $2 p^{5} 3 s 3 d$ & ${ }^{4} \mathrm{P}_{3 / 2}^{\circ}$ & & 58.05947 & 58.00538 & 58.10584 & $0.938(39)$ \\
\hline 40 & $2 p^{5} 3 s 3 d$ & ${ }^{4} \mathrm{~F}^{\circ}{ }_{9 / 2}^{3 / 2}$ & & 58.15573 & 58.09555 & 58.19396 & $1.000(40)$ \\
\hline 41 & $2 p^{5} 3 s 3 d$ & ${ }^{4} \mathrm{P}^{\circ}{ }_{5 / 2}^{9 / 2}$ & 58.25730 & 58.17918 & 58.11794 & 58.22565 & $0.799(41),-0.449(59)$ \\
\hline 42 & $2 p^{5} 3 p^{2}$ & ${ }^{2} \mathrm{~F}_{5 / 2}^{\circ}$ & & 58.19900 & 58.12751 & 58.21310 & $-0.641(42),-0.442(31)$ \\
\hline 43 & $2 p^{5} 3 p^{2}$ & ${ }^{4} \mathrm{D}^{\circ}{ }_{3 / 2}$ & & 58.21215 & 58.14156 & 58.23244 & $0.655(43),-0.410(37)$ \\
\hline 44 & $2 p^{5} 3 \mathrm{~s} 3 \mathrm{~d}$ & ${ }^{4} \mathrm{~F}^{\circ}{ }_{7 / 2}$ & & 58.21941 & 58.16102 & 58.25588 & $0.885(44)$ \\
\hline 45 & $2 p^{5} 3 p^{2}$ & ${ }^{2} \mathrm{~S}^{\circ}{ }_{1 / 2}$ & & 58.28129 & 58.21189 & 58.30146 & $0.712(45), 0.538(33), 0.447(36)$ \\
\hline 46 & $2 p^{5} 3 s 3 d$ & ${ }^{4} \mathrm{~F}^{\circ}{ }_{5 / 2}^{1 / 2}$ & 58.37577 & 58.30062 & 58.24386 & 58.33759 & $0.747(46), 0.431(50)$ \\
\hline 47 & $2 \mathrm{p}^{5} 3 \mathrm{~s}\left({ }^{1} \mathrm{P}\right) 3 \mathrm{~d}$ & ${ }^{2} \mathrm{D}^{\circ}{ }_{3 / 2}^{0 / 2}$ & & 58.40180 & 58.34352 & 58.43276 & $-0.576(58), 0.444(47)$ \\
\hline 48 & $2 p^{5} 3 p^{2}$ & ${ }^{4} \mathrm{~S}^{\circ}{ }_{3 / 2}^{3 / 2}$ & & 58.43197 & 58.36742 & 58.45592 & $0.479(48), 0.443(26), 0.437(52)$ \\
\hline 49 & $2 \mathrm{p}^{5} 3 \mathrm{~s} 3 \mathrm{~d}$ & ${ }^{4} \mathrm{D}^{\circ}{ }_{7 / 2}^{3 / 2}$ & 58.52157 & 58.45282 & 58.39564 & 58.48497 & $0.815(49), 0.497(61)$ \\
\hline 50 & $2 \mathrm{p}^{5} 3 \mathrm{~s}\left({ }^{1} \mathrm{P}\right) 3 \mathrm{~d}$ & ${ }^{2} \mathrm{~F}_{5 / 2}^{\circ}$ & 58.54891 & 58.48128 & 58.42242 & 58.51233 & $0.570(50), 0.482(59)$ \\
\hline 51 & $2 p^{5} 3 p^{2}\left({ }^{3} P\right)$ & ${ }^{2} \mathrm{D}^{\circ}{ }_{5 / 2}$ & & 58.51272 & 58.43827 & 58.53265 & $-0.673(51), 0.506(35)$ \\
\hline 52 & $2 p^{5} 3 p^{2}\left({ }^{1} S\right)$ & ${ }^{2} \mathrm{P}_{3 / 2}^{\circ}$ & & 58.51295 & 58.45493 & 58.54002 & $0.694(52)$ \\
\hline 53 & $2 \mathrm{p}^{5} 3 \mathrm{~s}\left({ }^{1} \mathrm{P}\right) 3 \mathrm{~d}$ & ${ }^{2} \mathrm{P}^{\circ}{ }_{1 / 2}^{3 / 2}$ & 58.53068 & 58.53541 & 58.48029 & 58.57178 & $0.622(53),-0.589(55)$ \\
\hline 54 & $2 \mathrm{p}^{5} 3 \mathrm{~s}\left({ }^{1} \mathrm{P}\right) 3 \mathrm{~d}$ & ${ }^{2} \mathrm{P}_{3 / 2}^{\circ}$ & 58.64915 & 58.71984 & 58.66234 & 58.74794 & $0.550(54),-0.548(56)$ \\
\hline 55 & $2 p^{5} 3 s 3 d$ & ${ }^{4} \mathrm{D}^{\circ}{ }_{1 / 2}$ & & 58.82057 & 58.76090 & 58.83807 & $0.646(55), 0.541(63),-0.420(67)$ \\
\hline 56 & $2 p^{5} 3 s 3 d$ & ${ }^{4} \mathrm{D}^{\circ}{ }_{3 / 2}$ & 58.98632 & 59.10868 & 59.04324 & 59.12486 & $-0.521(56),-0.405(65)$ \\
\hline 57 & $2 p^{5} 3 s\left({ }^{3} P\right) 3 d$ & ${ }^{2} \mathrm{~F}^{\circ}{ }_{7 / 2}$ & 58.73116 & 59.19215 & 59.13497 & 59.21341 & $0.772(57), 0.489(29),-0.402(61)$ \\
\hline 58 & $2 p^{5} 3 s 3 d$ & ${ }^{4} \mathrm{~F}_{3 / 2}^{\circ}$ & 59.25058 & 59.21520 & 59.14077 & 59.23393 & $0.700(58), 0.568(47)$ \\
\hline 59 & $2 p^{5} 3 s 3 d$ & ${ }^{4} \mathrm{D}^{\circ}{ }_{5 / 2}$ & 59.25058 & 59.23119 & 59.15303 & 59.24131 & $0.654(59), 0.535(46)$ \\
\hline 60 & $2 p^{5} 3 s\left({ }^{3} P\right) 3 d$ & ${ }^{2} \mathrm{D}^{\circ}{ }_{5 / 2}$ & 58.90430 & 59.27727 & 59.20474 & 59.28913 & $-0.653(60)$ \\
\hline 61 & $2 \mathrm{p}^{5} 3 \mathrm{~s}\left({ }^{1} \mathrm{P}\right) 3 \mathrm{~d}$ & ${ }^{2} \mathrm{~F}^{\circ} 7 / 2$ & 59.38727 & 59.33240 & 59.25137 & 59.33982 & $-0.666(61), 0.492(49), 0.461(44)$ \\
\hline 62 & $2 \mathrm{p}^{5} 3 \mathrm{~s}\left({ }^{1} \mathrm{P}\right) 3 \mathrm{~d}$ & ${ }^{2} \mathrm{D}^{\circ}{ }_{5 / 2}$ & 59.37816 & 59.40018 & 59.34082 & 59.42136 & $0.677(62)$ \\
\hline 63 & $2 p^{5} 3 p^{2}\left({ }^{3} P\right)$ & ${ }^{2} \mathrm{P}_{1 / 2}^{\circ}$ & & 59.40747 & 59.34593 & 59.43010 & $0.527(53),-0.482(27)$ \\
\hline 64 & $2 p^{5} 3 p^{2}\left({ }^{1} S\right)$ & ${ }^{2} \mathrm{P}_{1 / 2}^{\circ}$ & & 59.54384 & 59.47971 & 59.55638 & $0.881(64)$ \\
\hline
\end{tabular}


Table 1. continued.

\begin{tabular}{|c|c|c|c|c|c|c|c|}
\hline Index & Configuration & Level & NIST & GRASP $^{a}$ & GRASP $^{b}$ & $\mathrm{FAC}^{c}$ & Mixing coefficients $^{d}$ \\
\hline 65 & $2 \mathrm{p}^{5} 3 \mathrm{p}^{2}\left({ }^{3} \mathrm{P}\right)$ & ${ }^{2} \mathrm{P}_{3 / 2}^{\circ}$ & & 59.61159 & 59.55298 & 59.63031 & $0.601(65), 0.462(30)$ \\
\hline 66 & $2 p^{5} 3 s\left({ }^{3} P\right) 3 d$ & ${ }^{2} \mathrm{D}^{\circ}{ }_{3 / 2}$ & & 59.75825 & 59.68822 & 59.75204 & $-0.506(76), 0.506(66),-0.493(54)$ \\
\hline 67 & $2 \mathrm{p}^{5} 3 \mathrm{~s}\left({ }^{3} \mathrm{P}\right) 3 \mathrm{~d}$ & ${ }^{2} \mathrm{P}_{1 / 2}^{\circ}$ & 59.90670 & 60.02441 & 59.95160 & 60.00957 & $-0.802(67),-0.429(53)$ \\
\hline 68 & $2 p^{5} 3 p 3 d\left({ }^{3} S\right)$ & ${ }^{4} \mathrm{D}_{1 / 2}^{1 / 2}$ & & 60.09502 & 60.04611 & 60.14409 & $-0.730(68),-0.569(120)$ \\
\hline 69 & $2 p^{5} 3 p 3 d\left({ }^{3} S\right)$ & ${ }^{4} \mathrm{D}_{3 / 2}$ & & 60.17116 & 60.11829 & 60.21617 & $0.740(69), 0.517(119)$ \\
\hline 70 & $2 \mathrm{p}^{5} 3 \mathrm{~s}\left({ }^{3} \mathrm{P}\right) 3 \mathrm{~d}$ & ${ }^{2} \mathrm{~F}_{5 / 2}^{\circ}$ & 59.74267 & 60.20698 & 60.12842 & 60.20253 & $0.619(70), 0.407(60)$ \\
\hline 71 & $2 \mathrm{p}^{5} 3 \mathrm{p} 3 \mathrm{~d}\left({ }^{3} \mathrm{~S}\right)$ & ${ }^{4} \mathrm{D}_{5 / 2}$ & & 60.29006 & 60.23238 & 60.32960 & $0.759(71), 0.410(99)$ \\
\hline 72 & $2 \mathrm{p}^{5} 3 \mathrm{p} 3 \mathrm{~d}\left({ }^{3} \mathrm{D}\right)$ & ${ }^{4} \mathrm{G}_{7 / 2}$ & & 60.41131 & 60.35233 & 60.44618 & $0.616(72), 0.429(74), 0.403(82)$ \\
\hline 73 & $2 \mathrm{p}^{5} 3 \mathrm{p} 3 \mathrm{~d}$ & ${ }^{4} \mathrm{G}_{9 / 2}$ & & 60.43500 & 60.37302 & 60.47084 & $0.884(73)$ \\
\hline 74 & $2 p^{5} 3 p 3 d\left({ }^{3} S\right)$ & ${ }^{4} \mathrm{D}_{7 / 2}$ & & 60.44591 & 60.38502 & 60.50865 & $-0.681(74)$ \\
\hline 75 & $2 p^{5} 3 p 3 d$ & ${ }^{4} \mathrm{G}_{11 / 2}$ & & 60.47640 & 60.41011 & 60.47567 & $1.000(75)$ \\
\hline 76 & $2 \mathrm{p}^{5} 3 \mathrm{~s}\left({ }^{3} \mathrm{P}\right) 3 \mathrm{~d}$ & ${ }^{2} \mathrm{P}_{3 / 2}^{\circ}$ & 60.09806 & 60.47817 & 60.40230 & 60.47991 & $0.667(76), 0.408(66), 0.402(30)$ \\
\hline 77 & $2 \mathrm{p}^{5} 3 \mathrm{p} 3 \mathrm{~d}\left({ }^{3} \mathrm{D}\right)$ & ${ }^{2} \mathrm{D}_{5 / 2}$ & & 60.50269 & 60.44777 & 60.53828 & $-0.484(113)$ \\
\hline 78 & $2 \mathrm{p}^{5} 3 \mathrm{p} 3 \mathrm{~d}\left({ }^{3} \mathrm{D}\right)$ & ${ }^{2} \mathrm{D}_{3 / 2}$ & & 60.54189 & 60.48675 & 60.58196 & $-0.451(112), 0.428(78)$ \\
\hline 79 & $2 \mathrm{p}^{5} 3 \mathrm{p} 3 \mathrm{~d}\left({ }^{3} \mathrm{D}\right)$ & ${ }^{2} \mathrm{~F}_{7 / 2}$ & & 60.60902 & 60.54820 & 60.64577 & $0.680(79), 0.473(108)$ \\
\hline 80 & $2 \mathrm{p}^{5} 3 \mathrm{p} 3 \mathrm{~d}\left({ }^{1} \mathrm{P}\right)$ & ${ }^{2} \mathrm{~F}_{5 / 2}$ & & 60.61475 & 60.55605 & 60.64870 & $-0.559(101), 0.426(80)$ \\
\hline 81 & $2 \mathrm{p}^{5} 3 \mathrm{p} 3 \mathrm{~d}\left({ }^{1} \mathrm{P}\right)$ & ${ }^{2} \mathrm{P}_{1 / 2}$ & & 60.65721 & 60.60183 & 60.70206 & $-0.540(97), 0.509(81), 0.480(126)$ \\
\hline 82 & $2 \mathrm{p}^{5} 3 \mathrm{p} 3 \mathrm{~d}\left({ }^{1} \mathrm{D}\right)$ & ${ }^{2} \mathrm{G}_{7 / 2}$ & & 60.72887 & 60.67077 & 60.76248 & $0.531(82)$ \\
\hline 83 & $2 \mathrm{p}^{5} 3 \mathrm{p} 3 \mathrm{~d}\left({ }^{3} \mathrm{D}\right)$ & ${ }^{4} \mathrm{P}_{1 / 2}$ & & 60.78928 & 60.73533 & 60.83237 & $0.762(83), 0.531(115)$ \\
\hline 84 & $2 \mathrm{p}^{5} 3 \mathrm{p} 3 \mathrm{~d}\left({ }^{3} \mathrm{D}\right)$ & ${ }^{4} \mathrm{P}_{3 / 2}$ & & 60.82790 & 60.77171 & 60.86720 & $0.744(84),-0.550(86)$ \\
\hline 85 & $2 \mathrm{p}^{5} 3 \mathrm{p} 3 \mathrm{~d}\left({ }^{3} \mathrm{P}\right)$ & ${ }^{4} \mathrm{~F}_{9 / 2}$ & & 60.82979 & 60.76830 & 60.85897 & $0.615(85), 0.525(109), 0.500(91)$ \\
\hline 86 & $2 \mathrm{p}^{5} 3 \mathrm{p} 3 \mathrm{~d}\left({ }^{3} \mathrm{D}\right)$ & ${ }^{4} \mathrm{~S}_{3 / 2}$ & & 60.87238 & 60.81615 & 60.90864 & $0.609(86)$ \\
\hline 87 & $2 \mathrm{p}^{5} 3 \mathrm{p} 3 \mathrm{~d}\left({ }^{3} \mathrm{P}\right)$ & ${ }^{4} \mathrm{D}_{7 / 2}$ & & 60.88786 & 60.82874 & 60.92297 & $0.693(87),-0.464(94)$ \\
\hline 88 & $2 \mathrm{p}^{5} 3 \mathrm{p} 3 \mathrm{~d}\left({ }^{3} \mathrm{D}\right)$ & ${ }^{4} \mathrm{~F}_{5 / 2}$ & & 60.91229 & 60.85344 & 60.94462 & $-0.451(89), 0.406(114), 0.406(99)$ \\
\hline 89 & $2 \mathrm{p}^{5} 3 \mathrm{p} 3 \mathrm{~d}\left({ }^{3} \mathrm{D}\right)$ & ${ }^{4} \mathrm{P}_{5 / 2}$ & & 60.91779 & 60.85914 & 60.94992 & $0.742(89)$ \\
\hline 90 & $2 \mathrm{p}^{5} 3 \mathrm{p} 3 \mathrm{~d}\left({ }^{3} \mathrm{D}\right)$ & ${ }^{4} \mathrm{~F}_{3 / 2}$ & & 60.97310 & 60.91494 & 60.99975 & $-0.561(90)$ \\
\hline 91 & $2 \mathrm{p}^{5} 3 \mathrm{p} 3 \mathrm{~d}\left({ }^{3} \mathrm{D}\right)$ & ${ }^{4} \mathrm{~F}_{9 / 2}$ & & 61.01944 & 60.95573 & 61.04967 & $0.795(91),-0.591(85)$ \\
\hline 92 & $2 \mathrm{p}^{5} 3 \mathrm{p} 3 \mathrm{~d}\left({ }^{1} \mathrm{P}\right)$ & ${ }^{2} \mathrm{P}_{3 / 2}$ & & 61.02141 & 60.96448 & 61.05502 & $0.443(92)$ \\
\hline 93 & $2 \mathrm{p}^{5} 3 \mathrm{p} 3 \mathrm{~d}\left({ }^{1} \mathrm{P}\right)$ & ${ }^{2} \mathrm{D}_{5 / 2}$ & & 61.07479 & 61.01801 & 61.10393 & $-0.506(114), 0.433(93)$ \\
\hline 94 & $2 \mathrm{p}^{5} 3 \mathrm{p} 3 \mathrm{~d}\left({ }^{3} \mathrm{D}\right)$ & ${ }^{4} \mathrm{~F}_{7 / 2}$ & & 61.09930 & 61.03764 & 61.12961 & $0.513(94),-0.511(106), 0.429(121)$ \\
\hline 95 & $2 \mathrm{p}^{5} 3 \mathrm{p} 3 \mathrm{~d}\left({ }^{1} \mathrm{D}\right)$ & ${ }^{2} \mathrm{~F}_{5 / 2}$ & & 61.12272 & 61.06369 & 61.15376 & $0.610(95),-0.458(102)$ \\
\hline 96 & $2 \mathrm{p}^{5} 3 \mathrm{p} 3 \mathrm{~d}\left({ }^{3} \mathrm{D}\right)$ & ${ }^{4} \mathrm{D}_{7 / 2}$ & & 61.14633 & 61.08498 & 61.16957 & $0.826(96)$ \\
\hline 97 & $2 \mathrm{p}^{5} 3 \mathrm{p} 3 \mathrm{~d}\left({ }^{3} \mathrm{P}\right)$ & ${ }^{2} \mathrm{P}_{1 / 2}$ & & 61.16838 & 61.11025 & 61.19611 & $0.525(68),-0.486(120)$ \\
\hline 98 & $2 \mathrm{p}^{5} 3 \mathrm{p} 3 \mathrm{~d}\left({ }^{1} \mathrm{D}\right)$ & ${ }^{2} \mathrm{D}_{3 / 2}$ & & 61.23950 & 61.18234 & 61.26807 & $0.595(98),-0.507(119)$ \\
\hline 99 & $2 \mathrm{p}^{5} 3 \mathrm{p} 3 \mathrm{~d}\left({ }^{3} \mathrm{P}\right)$ & ${ }^{4} \mathrm{D}_{5 / 2}$ & & 61.26175 & 61.20205 & 61.28725 & -0.509 ( 99), $0.455(122)$ \\
\hline 100 & $2 \mathrm{p}^{5} 3 \mathrm{p} 3 \mathrm{~d}\left({ }^{1} \mathrm{P}\right)$ & ${ }^{2} \mathrm{D}_{3 / 2}$ & & 61.31016 & 61.24893 & 61.33183 & $-0.544(112), 0.484(100)$ \\
\hline 101 & $2 \mathrm{p}^{5} 3 \mathrm{p} 3 \mathrm{~d}\left({ }^{3} \mathrm{D}\right)$ & ${ }^{4} \mathrm{G}_{5 / 2}$ & & 61.32200 & 61.24585 & 61.33821 & $0.606(101), 0.502(107), 0.410(80)$ \\
\hline 102 & $2 p^{5} 3 p 3 d\left({ }^{3} P\right)$ & ${ }^{4} \mathrm{~F}_{5 / 2}$ & & 61.41068 & 61.34445 & 61.43000 & $0.451(80)$ \\
\hline 103 & $2 \mathrm{p}^{5} 3 \mathrm{p} 3 \mathrm{~d}\left({ }^{3} \mathrm{D}\right)$ & ${ }^{4} \mathrm{D}_{1 / 2}$ & & 61.41192 & 61.35321 & 61.42990 & $-0.573(103), 0.501(126)$ \\
\hline 104 & $2 \mathrm{p}^{5} 3 \mathrm{p} 3 \mathrm{~d}\left({ }^{3} \mathrm{P}\right)$ & ${ }^{4} \mathrm{~F}_{3 / 2}$ & & 61.47289 & 61.40208 & 61.49645 & $0.461(104), 0.411(100)$ \\
\hline 105 & $2 \mathrm{p}^{5} 3 \mathrm{p} 3 \mathrm{~d}\left({ }^{1} \mathrm{D}\right)$ & ${ }^{2} \mathrm{~S}_{1 / 2}$ & & 61.48154 & 61.42443 & 61.50666 & $-0.646(105),-0.628(115)$ \\
\hline 106 & $2 \mathrm{p}^{5} 3 \mathrm{p} 3 \mathrm{~d}\left({ }^{3} \mathrm{P}\right)$ & ${ }^{4} \mathrm{~F}_{7 / 2}$ & & 61.50415 & 61.42221 & 61.51326 & $0.605(72)$ \\
\hline 107 & $2 \mathrm{p}^{5} 3 \mathrm{p} 3 \mathrm{~d}\left({ }^{3} \mathrm{D}\right)$ & ${ }^{2} \mathrm{~F}_{5 / 2}$ & & 61.55458 & 61.47347 & 61.56445 & $-0.490(107), 0.466(125)$ \\
\hline 108 & $2 \mathrm{p}^{5} 3 \mathrm{p} 3 \mathrm{~d}\left({ }^{1} \mathrm{P}\right)$ & ${ }^{2} \mathrm{~F}_{7 / 2}$ & & 61.64063 & 61.56056 & 61.64670 & $0.499(87),-0.418(108), 0.414(106)$ \\
\hline 109 & $2 \mathrm{p}^{5} 3 \mathrm{p} 3 \mathrm{~d}\left({ }^{1} \mathrm{D}\right)$ & ${ }^{2} \mathrm{G}_{9 / 2}$ & & 61.66765 & 61.58175 & 61.66985 & $-0.662(109), 0.457(85), 0.445(73)$ \\
\hline 110 & $2 \mathrm{p}^{5} 3 \mathrm{p} 3 \mathrm{~d}\left({ }^{3} \mathrm{D}\right)$ & ${ }^{4} \mathrm{D}_{3 / 2}$ & & 61.68685 & 61.61493 & 61.69331 & $0.604(110)$ \\
\hline 111 & $2 \mathrm{p}^{5} 3 \mathrm{p} 3 \mathrm{~d}\left({ }^{3} \mathrm{D}\right)$ & ${ }^{2} \mathrm{G}_{9 / 2}$ & & 61.75682 & 61.69334 & 61.77370 & $0.919(111)$ \\
\hline 112 & $2 \mathrm{p}^{5} 3 \mathrm{p} 3 \mathrm{~d}\left({ }^{3} \mathrm{~S}\right)$ & ${ }^{2} \mathrm{D}_{3 / 2}$ & & 61.79240 & 61.72111 & 61.81103 & $0.588(90),-0.423(104)$ \\
\hline 113 & $2 \mathrm{p}^{5} 3 \mathrm{p} 3 \mathrm{~d}\left({ }^{3} \mathrm{~S}\right)$ & ${ }^{2} \mathrm{D}_{5 / 2}$ & & 61.86910 & 61.80037 & 61.88206 & $0.494(113), 0.439(118)$ \\
\hline 114 & $2 \mathrm{p}^{5} 3 \mathrm{p} 3 \mathrm{~d}\left({ }^{3} \mathrm{D}\right)$ & ${ }^{4} \mathrm{D}_{5 / 2}$ & & 61.94324 & 61.86516 & 61.94608 & $0.469(114), 0.439(93)$ \\
\hline 115 & $2 \mathrm{p}^{5} 3 \mathrm{p} 3 \mathrm{~d}\left({ }^{3} \mathrm{P}\right)$ & ${ }^{4} \mathrm{P}_{1 / 2}$ & & 61.95561 & 61.88979 & 61.96959 & $0.469(132),-0.466(83), 0.462(115)$ \\
\hline 116 & $2 p^{5} 3 p 3 d\left({ }^{3} P\right)$ & ${ }^{2} \mathrm{~F}_{7 / 2}$ & & 61.96033 & 61.89980 & 61.97643 & $-0.520(129), 0.496(116), 0.444(79)$ \\
\hline 117 & $2 p^{5} 3 p 3 d\left({ }^{3} P\right)$ & ${ }^{4} \mathrm{P}_{3 / 2}$ & & 61.99275 & 61.91457 & 61.99405 & $0.470(84), 0.456(123)$ \\
\hline 118 & $2 p^{5} 3 p 3 d\left({ }^{3} P\right)$ & ${ }^{4} \mathrm{P}_{5 / 2}$ & & 62.01950 & 61.94888 & 62.02740 & $0.494(118),-0.423(93)$ \\
\hline 119 & $2 p^{5} 3 p 3 d\left({ }^{3} P\right)$ & ${ }^{4} \mathrm{D}_{3 / 2}$ & & 62.05674 & 61.97802 & 62.06391 & $0.467(119)$ \\
\hline 120 & $2 \mathrm{p}^{5} 3 \mathrm{p} 3 \mathrm{~d}\left({ }^{3} \mathrm{P}\right)$ & ${ }^{4} \mathrm{D}_{1 / 2}^{3 / 2}$ & & 62.05790 & 61.97998 & 62.06754 & $-0.610(120),-0.444(81)$ \\
\hline 121 & $2 \mathrm{p}^{5} 3 \mathrm{p} 3 \mathrm{~d}\left({ }^{1} \mathrm{D}\right)$ & ${ }^{2} \mathrm{~F}_{7 / 2}^{1 / 2}$ & & 62.07990 & 61.99687 & 62.08230 & $-0.559(121)$ \\
\hline 122 & $2 \mathrm{p}^{5} 3 \mathrm{p} 3 \mathrm{~d}\left({ }^{1} \mathrm{D}\right)$ & ${ }^{2} \mathrm{D}_{5 / 2}$ & & 62.09892 & 62.02163 & 62.10258 & $0.499(130)$ \\
\hline 123 & $2 \mathrm{p}^{5} 3 \mathrm{p} 3 \mathrm{~d}\left({ }^{3} \mathrm{P}\right)$ & ${ }^{2} \mathrm{P}_{3 / 2}$ & & 62.11304 & 62.05018 & 62.11879 & $-0.492(123),-0.425(78),-0.418(127)$ \\
\hline 124 & $2 \mathrm{p}^{5} 3 \mathrm{p} 3 \mathrm{~d}\left({ }^{3} \mathrm{P}\right)$ & ${ }^{2} \mathrm{D}_{3 / 2}$ & & 62.34616 & 62.27680 & 62.32272 & $0.589(124), 0.457(78)$ \\
\hline 125 & $2 \mathrm{p}^{5} 3 \mathrm{p} 3 \mathrm{~d}\left({ }^{3} \mathrm{P}\right)$ & ${ }^{2} \mathrm{~F}_{5 / 2}$ & & 62.35587 & 62.28342 & 62.34591 & $-0.485(125), 0.446(122)$ \\
\hline 126 & $2 \mathrm{p}^{5} 3 \mathrm{p} 3 \mathrm{~d}\left({ }^{1} \mathrm{D}\right)$ & ${ }^{2} \mathrm{P}_{1 / 2}$ & & 62.39653 & 62.31859 & 62.38120 & $-0.549(97),-0.463(126)$ \\
\hline 127 & $2 \mathrm{p}^{5} 3 \mathrm{p} 3 \mathrm{~d}\left({ }^{1} \mathrm{D}\right)$ & ${ }^{2} \mathrm{P}_{3 / 2}$ & & 62.58476 & 62.50435 & 62.55824 & $0.492(127), 0.467(131)$ \\
\hline 128 & $2 \mathrm{p}^{5} 3 \mathrm{p} 3 \mathrm{~d}\left({ }^{3} \mathrm{D}\right)$ & ${ }^{2} \mathrm{P}_{1 / 2}$ & & 62.78038 & 62.70084 & 62.74799 & $-0.577(128), 0.520(132)$ \\
\hline
\end{tabular}


Table 1. continued.

\begin{tabular}{rlllllll}
\hline \hline Index & Configuration & Level & NIST & GRASP $^{a}$ & GRASP $^{b}$ & FAC $^{c}$ & Mixing coefficients $^{d}$ \\
\hline 129 & $2 \mathrm{p}^{5} 3 \mathrm{p} 3 \mathrm{~d}\left({ }^{3} \mathrm{D}\right)$ & ${ }^{2} \mathrm{G}_{7 / 2}$ & & 62.80742 & 62.72376 & 62.79937 & $0.656(129), 0.482(116)$ \\
130 & $2 \mathrm{p}^{5} 3 \mathrm{p} 3 \mathrm{~d}\left({ }^{3} \mathrm{P}\right)$ & ${ }^{2} \mathrm{D}_{5 / 2}$ & & 62.90893 & 62.83399 & 62.88355 & $0.533(130), 0.479(134)$ \\
131 & $2 \mathrm{p}^{5} 3 \mathrm{p} 3 \mathrm{~d}\left({ }^{3} \mathrm{D}\right)$ & ${ }^{2} \mathrm{P}_{3 / 2}$ & 62.93802 & 62.86270 & 62.92609 & $0.526(124),-0.494(131)$ \\
132 & $2 \mathrm{p}^{5} 3 \mathrm{p} 3 \mathrm{~d}\left({ }^{3} \mathrm{D}\right)$ & ${ }^{2} \mathrm{~S}_{1 / 2}$ & 63.11751 & 63.03638 & 63.09278 & $0.630(132), 0.574(128)$ \\
133 & $2 \mathrm{p}^{5} 3 \mathrm{p} 3 \mathrm{~d}\left({ }^{1} \mathrm{~S}\right)$ & ${ }^{2} \mathrm{D}_{3 / 2}$ & 63.27350 & 63.21048 & 63.29593 & $0.875(133)$ \\
134 & $2 \mathrm{p}^{5} 3 \mathrm{p} 3 \mathrm{~d}\left({ }^{1} \mathrm{~S}\right)$ & ${ }^{2} \mathrm{D}_{5 / 2}$ & 63.34064 & 63.27358 & 63.37006 & $0.850(134)$ \\
\hline
\end{tabular}

NIST: http://physics.nist.gov/PhysRefData.

${ }^{a}$ energies without Breit and QED corrections;

${ }^{b}$ energies with Breit and QED corrections;

${ }^{c}$ energies from the Flexible Atomic Code of Gu (2003);

${ }^{d}$ mixing coefficient of the level (in bracket).

paper we report our results for energy levels and radiative rates ( $A$-values) for five types of transitions, namely electric dipole (E1), electric quadrupole (E2), magnetic dipole (M1), magnetic quadrupole (M2) and electric octupole (E3), as all of these are required in the modelling of plasmas. Additionally, we report lifetimes for all excited levels, although no measurements are presently available with which to compare these results.

\section{Energy levels}

The $2 \mathrm{p}^{6} 3 \ell, 2 \mathrm{p}^{5} 3 \mathrm{~s}^{2}, 2 \mathrm{p}^{5} 3 \mathrm{~s} 3 \mathrm{p}, 2 \mathrm{p}^{5} 3 \mathrm{~s} 3 \mathrm{~d}, 2 \mathrm{p}^{5} 3 \mathrm{p} 3 \mathrm{~d}$, and $2 \mathrm{p}^{5} 3 \mathrm{p}^{2}$ configurations of FeXVI give rise to 134 fine-structure levels, listed in Table 1 . Our calculated energies obtained from the GRASP code, with and without including Breit and QED effects, are given in this table along with those from the experimental compilations of NIST (http://physics.nist.gov/PhysRefData). For our calculations, we have used the option of extended average level $(\mathrm{EAL}$ ), in which a weighted (proportional to $2 j+1$ ) trace of the Hamiltonian matrix is minimized. This produces a compromise set of orbitals describing closely lying states with moderate accuracy. The inclusion of Breit and QED effects lowers the energies up to a maximum of $0.08 \mathrm{Ryd}(\leq 0.2 \%)$ - see for example, levels 61, 101 and 131. In general, the theoretical energies agree well in magnitude with the experimental ones, but differences are up to $0.4 \mathrm{Ryd}(\leq 0.7 \%)$ for some levels, such as: 11 , 57 and 70. Additionally, our energies without the Breit and QED corrections are comparatively in better agreement with the corresponding experimental results, although the latter are available for only a few levels.

However, the level orderings in theory and experiment are slightly different in a few instances, such as for levels: 11/12 and 61/62. Most of these levels have energies very close to each other, and differing amount of configuration interaction (CI) may result in slightly different level orderings. However, a more serious problem is of level indentification. Some of the levels of a configuration with the same $J$ value are highly mixed, such as levels $11 / 17\left(2 \mathrm{p}^{5} 3 \mathrm{~s} 3 \mathrm{p}^{2} \mathrm{D}_{3 / 2},{ }^{4} \mathrm{D}_{3 / 2}\right), 26 / 30\left(2 \mathrm{p}^{5} 3 \mathrm{p}^{2}{ }^{4} \mathrm{P}_{3 / 2}^{\circ},{ }^{2} \mathrm{P}_{3 / 2}^{\circ}\right)$ and $66 / 76\left(2 \mathrm{p}^{5} 3 \mathrm{~s} 3 \mathrm{~d}^{2} \mathrm{D}_{3 / 2}^{\circ},{ }^{2} \mathrm{P}_{3 / 2}^{\circ}\right)$. For these (and other similar) levels the identification of a particular level is not unique, and is not based on the strength of the dominant eigenvector alone. This is a common problem in all atomic structure calculations, especially when the levels of a configuration are highly mixed, and the addition of more CI complicates the identifications further rather than making the task easier. Therefore, the configuration and the $J$ values given in Table 1 are definite, but the corresponding $L S J$ designation provided for a level is only for guidance, and is liable to interchange. This is the main reason that some of the experimental levels, such as $2 \mathrm{p}^{5} 3 \mathrm{~s}\left({ }^{1} \mathrm{P}\right) 3 \mathrm{p}^{2} \mathrm{D}_{3 / 2}$ and $2 p^{5} 3 s\left({ }^{3} P\right) 3 p{ }^{2} D_{3 / 2}$, i.e. levels 11 and 24 , appear to be listed at the wrong places.

We have taken all precautions to label the levels in Table 1, although some ambiguity in their designations cannot be avoided as discussed above. Furthermore, in the absence of any other theoretical or experimental energies to compare with, we have performed another calculation from the Flexible Atomic Code (FAC) of $\mathrm{Gu}$ (2003), which is easily available from the website http://kipac-tree.stanford.edu/fac. Energy levels obtained from this code are also listed in Table 1, and the excellent agreement (within 0.1 Ryd) between the GRASP and FAC energy levels gives confidence in our results. The level orderings are also (nearly) the same in both calculations, although some closely spaced levels, such as 41/42, 69/70 and 102/103, have switched positions. Finally, in the last column of Table 1 we have included the mixing coefficients (largest three where appropriate), which may be helpful for further comparisons and redesignations of the levels.

\section{Radiative rates}

The absorption oscillator strength $\left(f_{i j}\right)$ and radiative rate $A_{j i}$ (in $\mathrm{s}^{-1}$ ) for a transition $i \rightarrow j$ are related by the following expression:

$f_{i j}=\frac{m c}{8 \pi^{2} e^{2}} \lambda_{j i}^{2} \frac{\omega_{j}}{\omega_{i}} A_{j i}=1.49 \times 10^{-16} \lambda_{j i}^{2}\left(\omega_{j} / \omega_{i}\right) A_{j i}$

where $m$ and $e$ are the electron mass and charge, respectively, $c$ is the velocity of light, $\lambda_{j i}$ is the transition energy/wavelength in $\AA$, and $\omega_{i}$ and $\omega_{j}$ are the statistical weights of the lower $(i)$ and upper $(j)$ levels, respectively. Similarly, the oscillator strength $f_{i j}$ (dimensionless) and the line strength $\mathrm{S}$ (in atomic unit, 1 a.u. = $6.460 \times 10^{-36} \mathrm{~cm}^{2} \mathrm{esu}^{2}$ ) are related by the following standard equations:

for the electric dipole (E1) transitions:

$A_{j i}=\frac{2.0261 \times 10^{18}}{\omega_{j} \lambda_{j i}^{3}} S^{\mathrm{E} 1} \quad$ and $\quad f_{i j}=\frac{303.75}{\lambda_{j i} \omega_{i}} S^{\mathrm{E} 1}$,

for the magnetic dipole (M1) transitions:

$A_{j i}=\frac{2.6974 \times 10^{13}}{\omega_{j} \lambda_{j i}^{3}} S^{\mathrm{M} 1} \quad$ and $\quad f_{i j}=\frac{4.044 \times 10^{-3}}{\lambda_{j i} \omega_{i}} S^{\mathrm{M} 1}$

for the electric quadrupole (E2) transitions:

$A_{j i}=\frac{1.1199 \times 10^{18}}{\omega_{j} \lambda_{j i}^{5}} S^{\mathrm{E} 2} \quad$ and $\quad f_{i j}=\frac{167.89}{\lambda_{j i}^{3} \omega_{i}} S^{\mathrm{E} 2}$, 
Table 4. Transition wavelengths $\left(\lambda_{i j}\right.$ in $\AA$ ), radiative rates $\left(A_{j i}\right.$ in $\left.\mathrm{s}^{-1}\right)$, and oscillator strengths $\left(\mathrm{f}_{i j}\right.$, dimensionless) for some $\left(f \geq 10^{-8}\right)$ magnetic dipole (M2) and electric octupole (E3) transitions of Fe XVI. $\left(a \pm b \equiv a \times 10^{ \pm b}\right)$.

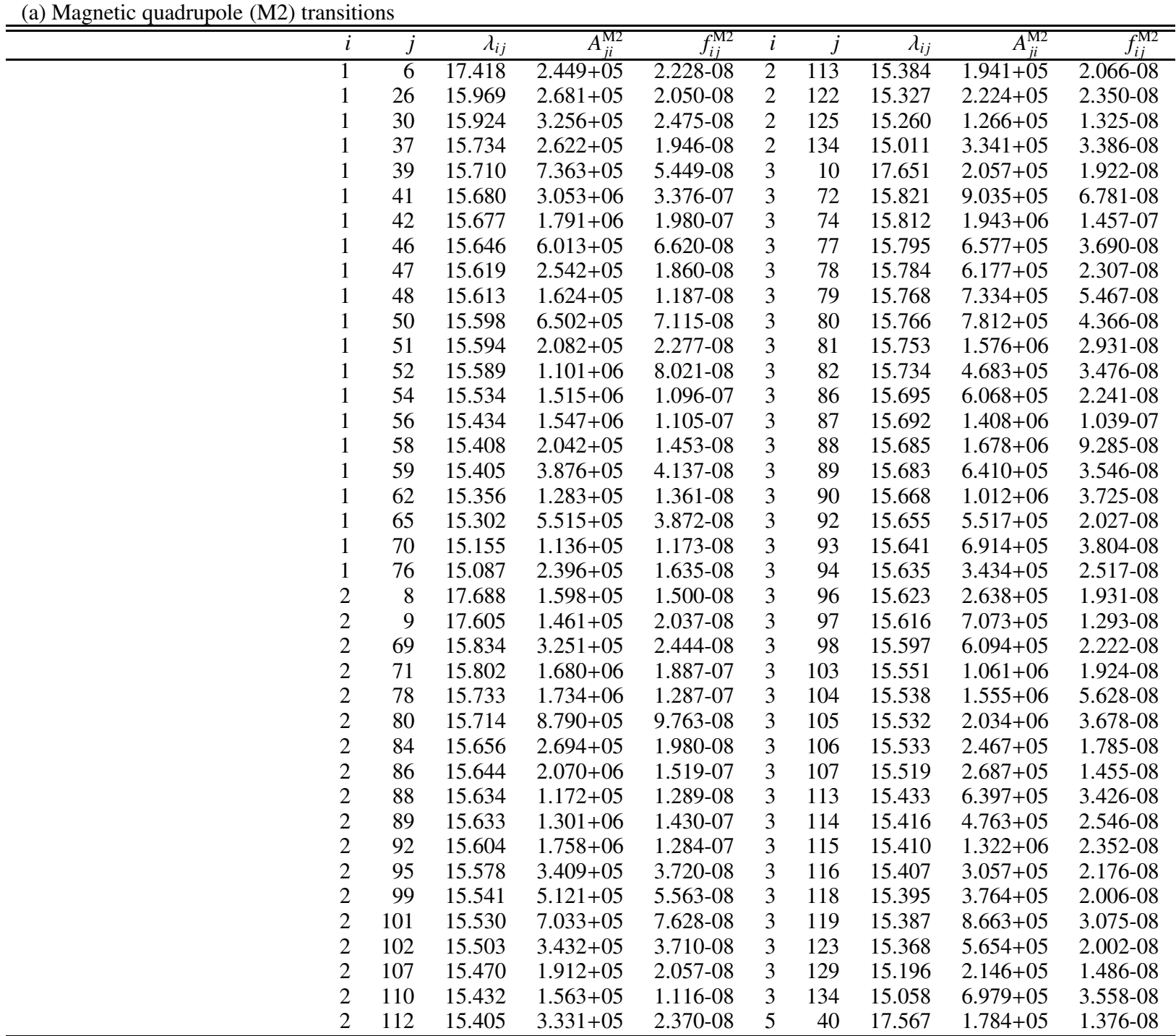

(b) Electric octupole (E3) transitions

\begin{tabular}{rrrrrrrrrr}
\hline$i$ & $j$ & $\lambda_{i j}$ & $A_{j i}^{\mathrm{E} 3}$ & $f_{i j}^{\mathrm{E} 3}$ & $i$ & $j$ & $\lambda_{i j}$ & $A_{j i}^{\mathrm{E} 3}$ & $f_{i j}^{\mathrm{E} 3}$ \\
\hline 1 & 44 & 15.668 & $9.998+04$ & $1.472-08$ & 2 & 107 & 15.470 & $9.715+04$ & $1.046-08$ \\
1 & 46 & 15.646 & $2.078+05$ & $2.288-08$ & 2 & 113 & 15.384 & $2.569+05$ & $2.735-08$ \\
1 & 49 & 15.605 & $2.649+05$ & $3.869-08$ & 2 & 121 & 15.333 & $1.483+05$ & $2.091-08$ \\
1 & 50 & 15.598 & $3.122+05$ & $3.416-08$ & 2 & 134 & 15.011 & $1.183+05$ & $1.199-08$ \\
1 & 57 & 15.410 & $2.348+05$ & $3.344-08$ & 3 & 82 & 15.734 & $1.466+05$ & $1.088-08$ \\
1 & 59 & 15.405 & $1.503+05$ & $1.604-08$ & 3 & 85 & 15.708 & $2.682+05$ & $2.481-08$ \\
1 & 60 & 15.392 & $1.117+05$ & $1.190-08$ & 3 & 95 & 15.628 & $2.140+05$ & $1.175-08$ \\
1 & 61 & 15.380 & $4.066+05$ & $5.767-08$ & 3 & 96 & 15.623 & $1.892+05$ & $1.384-08$ \\
1 & 70 & 15.155 & $1.417+05$ & $1.464-08$ & 3 & 100 & 15.579 & $3.972+05$ & $1.445-08$ \\
2 & 72 & 15.770 & $1.314+05$ & $1.959-08$ & 3 & 102 & 15.553 & $2.398+05$ & $1.305-08$ \\
2 & 74 & 15.761 & $1.731+05$ & $2.578-08$ & 3 & 109 & 15.491 & $2.891+05$ & $2.600-08$ \\
2 & 77 & 15.744 & $1.067+05$ & $1.190-08$ & 3 & 111 & 15.461 & $3.844+05$ & $3.444-08$ \\
2 & 82 & 15.683 & $1.827+05$ & $2.695-08$ & 3 & 121 & 15.382 & $2.578+05$ & $1.829-08$ \\
2 & 93 & 15.590 & $2.410+05$ & $2.634-08$ & 3 & 122 & 15.376 & $3.466+05$ & $1.843-08$ \\
2 & 106 & 15.483 & $2.385+05$ & $3.428-08$ & 3 & 129 & 15.196 & $1.467+05$ & $1.016-08$ \\
\hline
\end{tabular}

and for the magnetic quadrupole (M2) transitions:

$A_{j i}=\frac{1.4910 \times 10^{13}}{\omega_{j} \lambda_{j i}^{5}} S^{\mathrm{M} 2} \quad$ and $\quad f_{i j}=\frac{2.236 \times 10^{-3}}{\lambda_{j i}^{3} \omega_{i}} S^{\mathrm{M} 2}$.
In Table 2 we present transition energies/wavelengths ( $\lambda$ in $\AA$ ), radiative rates $\left(A_{j i}\right.$ in $\left.\mathrm{s}^{-1}\right)$, oscillator strengths $\left(f_{i j}\right.$, dimensionless), and line strengths ( $S$ in a.u.), in length form only, for all 2676 electric dipole (E1) and 3418 magnetic quadrupole (M2) transitions among the 134 levels of Fe XVI. The indices used 
to represent the lower and upper levels of a transition have already been defined in Table 1. Similar results for 3942 electric quadrupole (E2) and 3023 magnetic dipole (M1) transitions are listed in Table 3. Furthermore, sometimes the electric octupole (E3) transitions are also observed, and are required in plasma modelling. Therefore, the $A$ - and $f$-values for all 3187 E3 transitions are also listed in Table 2. However, a majority of the E3 (and M2) transitions have very small oscillator strengths. Therefore, in Table 4 we have listed the $A$ - and $f$-values of all those $\mathrm{M} 2$ and $\mathrm{E} 3$ transitions, whose $f$-values are $\geq 10^{-8}$. Since no other similar results are available in the literature, we hope that the presently reported $A$-values for all types of transitions will be useful in plasma modelling.

In the absence of other similar $A$-values being available in the literature with which to compare, it is difficult to assess the accuracy of our present results. A general criterion to assess the accuracy is to compare the length and velocity forms of the $f$ - or $A$-values. Before we discuss these we would like to remind readers that such comparisons are only desirable, but are not a fully sufficient test to assess accuracy, as different calculations (or combinations of configurations) may give comparable $f$-values in the two forms, but entirely different results in magnitude - see Aggarwal et al. (2007) for further details and comparisons.

Since the electric dipole (E1) transitions are comparatively more important, we focus on the accuracy assessments of their $A$-values alone. Among the stronger transitions (i.e. $f \geq 0.01$ ), the length and velocity forms differ by over $20 \%$ for $267(10 \%)$ transitions, whereas only $49(2 \%)$ transitions have differing $A$-values of over $50 \%$. Among these, four transitions, namely $25-66,57-118,65-115$ and $66-128$, have the ratio $A_{\mathrm{L}} / A_{\mathrm{V}}=$ $f_{\mathrm{L}} / f_{\mathrm{V}}$ with values of up to 10 . Therefore, based on a satisfactory agreement between the two forms, we may state that the accuracy of our listed $A$-values is better than $20 \%$ for a majority of the strong transitions. Differences between the two forms for weaker transitions $(f<0.01)$ can sometimes be several orders of magnitude. Particularly noteworthy are 5 transitions, namely 27-155 $\left(f \sim 10^{-7}\right), 38-81\left(f \sim 10^{-8}\right)$, 65-99 $\left(f \sim 10^{-6}\right)$, $69-70\left(f \sim 10^{-7}\right)$, and $76-120\left(f \sim 10^{-8}\right)$, for which the ratio $A_{\mathrm{L}} / A_{\mathrm{V}}$ is very large. However, all such transitions are very weak, and hence sensitive to mixing coefficients, but do not affect the overall accuracy of the calculations. Before concluding we make one more comparison with our calculations from FAC.

A comparison made between the $A$-values from GRASP and FAC (not listed in Table 2) indicates excellent agreement for a majority of the strong transitions. However, $143(5 \%)$ of the transitions have $f$-values differing by over $20 \%$, whereas only $42(1.6 \%)$ transitions differ by over $50 \%$. Among these, only three transitions, namely $34-88,35-88$ and $41-88$, have the $f$ values differing by an order of magnitude. Considering that the calculations have been performed for a large number of transitions, such differences for a few transitions do not affect the overall accuracy of our results, and we may state with confidence that our listed $A$-values are probably accurate to better than $20 \%$ for a majority of the strong transitions.

\section{Lifetimes}

The lifetime $\tau$ for a level $j$ is defined as follows:

$$
\tau_{j}=\frac{1}{\sum_{i} A_{j i}} .
$$

Since this is a measurable parameter, it provides a check on the accuracy of the calculations. However, to our knowledge no measurement of lifetimes for the levels of Fe XVI are so far available in the literature. Nevertheless, in Table 5 we list our calculated lifetimes, which include the contributions from four types of transitions, i.e. E1, E2, M1 and M2. These results may be helpful for future comparisons with theory or experiments. Most of the $\tau$ values are very small ( $\sim p s)$, but the lifetime of the $2 \mathrm{p}^{5} 3 \mathrm{~s} 3 \mathrm{p}{ }^{4} \mathrm{D}_{7 / 2}$ level is $\sim \mu \mathrm{s}$, and therefore it may be comparatively easier to measure.

\section{Conclusions}

In the present work, results for energy levels, radiative rates, oscillator strengths, and line strengths for all transitions among the lowest 134 levels of Fe XVI have been presented. Additionally, results for radiative rates have been presented for five types of transitions, namely E1, E2, E3, M1 and M2. A complete set of results are likely to be useful for the modelling of a variety of plasmas.

Based on the comparison made between the length and velocity forms of the oscillator strengths, as well as the comparison between the GRASP and FAC calculations, we assess that our radiative rates are accurate to better than $20 \%$ for a majority of strong transitions. Similarly, based on the comparison between the GRASP and FAC calculations, as well as with the available compiled experimental results, our energy levels are assessed to be accurate to better than $1 \%$. However, the level designations are likely to fluctuate for a few of them, for which further work is recommended.

Lifetimes for all excited levels of Fe XVI are listed, but no comparisons could be possible due to the paucity of other theoretical or experimental results.

Our calculations for collision strengths, and subsequently for excitation rates, are in progress. These calculations, being highly computationally demanding, will take a considerably long time to conclude. We hope to present our results for these highly desired and important parameters in future.

Acknowledgements. This work has been financed by the Engineering and Physical Sciences and Particle Physics and Astronomy Research Councils of the United Kingdom, and FPK is grateful to AWE Aldermaston for the award of a William Penney Fellowship. Finally, we thank an anonymous referee for a critical reading of the earlier version of the paper, and for providing some constructive comments.

\section{References}

Aggarwal, K. M., \& Keenan, F. P. 2006, A\&A, 450, 1249

Aggarwal, K. M., Tayal, V., Gupta, G. P., \& Keenan, F. P. 2007, ADNDT, in press

Bautista, M. A. 2000, J. Phys. B, 33, 71

Berrington, K. A., Eissner, W. B., \& Norrington, P. H. 1995, Comput. Phys. Commun., 92, 290

Cornille, M., Dubau, J., Faucher, P., Bely-Dubau, F., \& Blancard, C. 1994, A\&AS, 105, 77

Dere, K. P., Landi, E., Young, P. R., \& Del Zanna, G. 2001, ApJS, 134, 331

Dyall, K. G., Grant, I. P., Johnson, C. T., Parpia, F. A., \& Plummer, E. P. 1989, Comput. Phys. Commun., 55, 424

Eissner, W., \& Seaton, M. J. 1972, J. Phys. B, 5, 2187

Eissner, W., Jones, M., \& Nussbaumer, H. 1974, Comput. Phys. Commun., 8, 270

Gu, M. F. 2003, ApJ, 582, 1241

Norrington, P. H., \& Grant, I. P. 2007, Comput. Phys. Commun., in preparation 